\title{
Measuring the impact of a quality improvement collaboration to decrease maternal mortality in a Ghanaian regional hospital ${ }^{2}$
}

\author{
Emmanuel K. Srofenyoh ${ }^{\text {a,* }}$, Nicholas J. Kassebaum ${ }^{\text {b }}$, David M. Goodman ${ }^{\text {, }}$, \\ Adeyemi J. Olufolabi ${ }^{\mathrm{d}}$, Medge D. Owen ${ }^{\mathrm{e}}$ \\ a Ridge Regional Hospital, Ghana Health Service, Accra, Ghana \\ ${ }^{\mathrm{b}}$ Department of Anesthesiology and Pain Medicine, and the Institute for Health Metrics and Evaluation, Seattle Children's Hospital, University of Washington, Seattle, WA, USA \\ ' Department of Obstetrics and Gynecology, Wake Forest School of Medicine, Winston-Salem, NC, USA \\ d Department of Anesthesiology, Duke University School of Medicine, Durham, NC, USA \\ e Department of Anesthesiology, Wake Forest School of Medicine, Winston-Salem, NC, USA
}

\section{A R T I C L E I N F O}

\section{Article history:}

Received 3 September 2015

Received in revised form 24 November 2015

Accepted 1 April 2016

\section{Keywords:}

Case fatality rates

Maternal mortality

Quality improvement

Risk adjustment

Sub-Saharan Africa

\begin{abstract}
A B S T R A C T
Objective: To evaluate the performance of a continuous quality improvement collaboration at Ridge Regional Hospital, Accra, Ghana, that aimed to halve maternal and neonatal deaths. Methods: In a quasi-experimental, pre- and post-intervention analysis, system deficiencies were analyzed and 97 improvement activities were implemented from January 2007 to December 2011. Data were collected on outcomes and implementation rates of improvement activities. Severity-adjustment models were used to calculate counterfactual mortality ratios. Regression analysis was used to determine the association between improvement activities, staffing, and maternal mortality. Results: Maternal mortality decreased by 22.4\% between 2007 and 2011, from 496 to 385 per 100000 deliveries, despite a 50\% increase in deliveries and five- and three-fold increases in the proportion of pregnancies complicated by obstetric hemorrhage and hypertensive disorders of pregnancy, respectively. Case fatality rates for obstetric hemorrhage and hypertensive disorders of pregnancy decreased from $14.8 \%$ to $1.6 \%$ and $3.1 \%$ to $1.1 \%$, respectively. The mean implementation score was $68 \%$ for the 97 improvement processes. Overall, 43 maternal deaths were prevented by the intervention; however, risk severity-adjustment models indicated that an even greater number of deaths was averted. Mortality reduction was correlated with 26 continuous quality improvement activities, and with the number of anesthesia nurses and labor midwives. Conclusion: The implementation of quality improvement activities was closely correlated with improved maternal mortality.

(c) 2016 International Federation of Gynecology and Obstetrics. Published by Elsevier Ireland Ltd. All rights reserved.
\end{abstract}

\section{Introduction}

As 2015 concluded, low-income countries were still working to achieve their Millennium Development Goal targets. Nevertheless, Millennium Development Goal 5, which aimed to reduce maternal mortality by $75 \%$ between 1990 and 2015, was difficult to attain for reasons including poor access to quality care [1-3]. In 2007, the WHO Framework for Action [4] identified "quality of health services" as important in improving health outcomes. Some countries have succeeded in reducing maternal mortality, citing strengthened clinical and organizational management, as well as quality improvement mechanisms as contributing factors [5].

\footnotetext{
is Data presented in part at the Society for Obstetric Anesthesiology and Perinatology 45th Annual Meeting; April 24-28, 2013; San Juan, Puerto Rico: Abstract \#BP4.

* Corresponding author at: Ridge Regional Hospital, PO Box 473, Accra, Ghana. Tel.: + 233208115809.

E-mail address: emmanuelsrofenyoh@gmail.com (E.K. Srofenyoh).
}

Continuous quality improvement (CQI) programs are being increasingly implemented worldwide, with variable effectiveness [6-9]. Primary outcomes can be used to measure health interventions, but outcomes alone do not define quality care. Process indicators may demonstrate that quality improvement precedes change in outcome, but the lack of standardized measurement criteria has limited the ability to assess process methods [10]. The strength of the association between the process indicator and the outcome of interest must be examined when process indicators are being used to measure healthcare delivery.

Kybele, Inc. and the Ghana Health Service began a 5-year collaboration in 2007 at Ridge Regional Hospital (RRH), Accra, to reduce the number of maternal and neonatal deaths by half. Kybele is a nonprofit humanitarian organization that promotes safe childbirth through innovative partnerships (http://www.kybeleworldwide.org). RRH is the largest obstetric referral center within the Ghana Health Service, with a 90 -bed maternity unit providing comprehensive care. The role of referral hospitals is to manage complicated pregnancies. As such, approximately $70 \%$ of deliveries at RRH are high-risk prenatal or peripartum referrals. Thus, the extent to which these institutions are supported 
and developed to manage complex cases will affect the national effort of reducing maternal mortality.

The present study evaluated the performance of the collaboration at RRH by analyzing maternal mortality and the statistical correlation between implementation of CQI activities, staffing, and clinical outcomes. A conceptual framework for the design, implementation, and evaluation of a CQI program aimed at reducing maternal mortality in referral centers in low-resource settings is proposed.

\section{Materials and methods}

A full description of the methods used in the present study has been previously published [7]. The collaboration was led by Ghanaian clinicians and supported by triannual visits from healthcare professionals from the USA, Canada, and the UK to provide coaching and direction. Patient care processes were analyzed consecutively by joint RRH-Kybele teams using immersive, interactive, and consultative methods. The initial evaluation revealed numerous systems-level challenges, including poor staff communication, late referrals of high-risk patients, infrequent maternal assessment, limited neonatal resuscitation, few standardized protocols, poorly maintained equipment, treatment delay, and minimal blood product availability. Solutions were proposed by the multidisciplinary team and incorporated into a strategic template or "process map." For each deficiency, assessment metrics and an implementation and advocacy plan were determined and traceable outputs and outcomes were established. The process evolved into an institutional Framework of Change Model for reducing perinatal mortality as previously described [7].

The present evaluation represents a quasi-experimental pre- and post-intervention analysis. The Ghana Health Service directorate granted permission for assessment of interventions conducted during the project. Because no patient-level data were collected, informed consent was not required. Data collection was initially based on manual abstraction from "admission and discharge" and "delivery" ledgers. In 2009, an electronic database was established to support and validate manual abstraction. Data on obstetric outcomes included total deliveries, maternal deaths, and the caseload of obstetric hemorrhage $(\mathrm{OH})$ and hypertensive disorders of pregnancy (HDoP).

HDoP was defined as pregnancy-induced hypertension $(\geq 140$ / $90 \mathrm{~mm} \mathrm{Hg}$ after 20 weeks of pregnancy without proteinuria), preeclampsia (hypertension $\geq 140 / 90 \mathrm{~mm} \mathrm{Hg}$ after 20 weeks of pregnancy with proteinuria $\geq 1+$ on dipstick), superimposed pre-eclampsia (worsening hypertension with new-onset proteinuria), imminent eclampsia (pre-eclampsia with headache, epigastric pain, nausea, or blurred vision), and eclampsia. Cases classified as $\mathrm{OH}$ included primary postpartum hemorrhage ( $\geq 500 \mathrm{~mL}$ for vaginal or $\geq 1000 \mathrm{~mL}$ for cesarean delivery within 24 hours of delivery), secondary postpartum hemorrhage (bleeding $>24$ hours after delivery), prepartum hemorrhage due to placenta previa, abruption placenta, and ruptured uterus.

The 97 CQI activities were grouped within three bundles (Personnel [P], Quality-communication [Q], and Systems-management [S]), and nine sub-bundles (Table 1 ). This categorization was based on the broad goals of the various improvement activities. Implementation of these activities was led by local clinicians with support and mentorship from the external collaborators. The implementation strategy included triannual visits of the external teams, prioritization of improvement activities, data collection, and implementation.

Three assessors (E.K.S., A.J.O., and M.D.O.) independently assigned implementation scores for the 97 improvement processes three times per year. Scores were assigned in quartiles ranging from $0 \%$ to $100 \%$, color coded, and displayed in the hospital. Objectives that had numerical outcomes could be assessed quantitatively, but other assessments were subjective and based on the evaluators' in-depth knowledge of the system. To limit subjectivity, disagreements between evaluators were resolved through discussion so that one score for each item was recorded. Mean implementation scores for each improvement activity,
Table 1

Classification of bundles of improvement activities.

\begin{tabular}{cl}
\hline $\begin{array}{l}\text { Bundle and } \\
\text { sub-bundle }\end{array}$ & Activity \\
\hline P & \\
P1 & Leadership and organizational development \\
P2 & Motivation, empowerment, responsibility, and accountability of staff \\
P3 & Improvement of knowledge and skills \\
Q & Improvement of service quality and standards of clinical care \\
Q1 & Facilitation of communication between and within departments \\
Q2 & Improvement of communication and feedback with referring institutions \\
Q3 & Improvement of patient flow processes and timeliness of care \\
S & Improvement of physical workspace and maximization of capacity \\
S1 & Improvement of resources (staffing and equipment) and logistics \\
S2 & supplies \\
S3 &
\end{tabular}

Abbreviations: P, personnel bundle; Q quality-communication bundle; S, systemsmanagement bundle.

sub-bundle, and bundle were calculated in addition to an overall "PQS score" that summarized implementation of the entire change model.

Two counterfactual clinical scenarios were developed as severity adjustments to account for the evolving case-mix over the study period (Fig. 1). We used prevalence and case fatality rate (CFR) data for $\mathrm{OH}$ and HDoP to calculate CFR and prevalence for other contributing conditions using the following calculation:

$C F R_{\text {total }}=\left(C F R_{\text {hem }} \times\right.$ Prev $\left._{\text {hem }}\right)+\left(C F R_{\text {htn }} \times\right.$ Prev $\left._{\text {htn }}\right)+\left(C F R_{\text {other }} \times\right.$ Prev $\left._{\text {other }}\right)$

This assumes that the overall CFR of the total number of cases referred or delivered at the hospital is dependent on the sum of causespecific CFR and the prevalence of contributing conditions. In the first counterfactual scenario (CF1), all prevalence rates were held constant at their observed 2006 values and paired with observed values for CFR at each timepoint. In the second counterfactual scenario (CF2), CFR values were held constant at those observed in 2006, but paired with time-specific prevalence values. Because the proportions of the most severe forms of the two complications did not change significantly (e.g. eclampsia consistently contributed to $16 \%-17 \%$ of $\mathrm{HDoP}$ ), further severity adjustments were unnecessary.

It was hypothesized that change could be due to the implementation of improvement activities and/or staffing. To determine which factors most closely correlated with changes in mortality, an ordinary least squares (OLS) linear regression [11] was performed, including CFR for all deliveries, $\mathrm{HDoP}, \mathrm{OH}$, and the remaining mortalities for the observed and counterfactual scenarios versus annual mean CQI activity

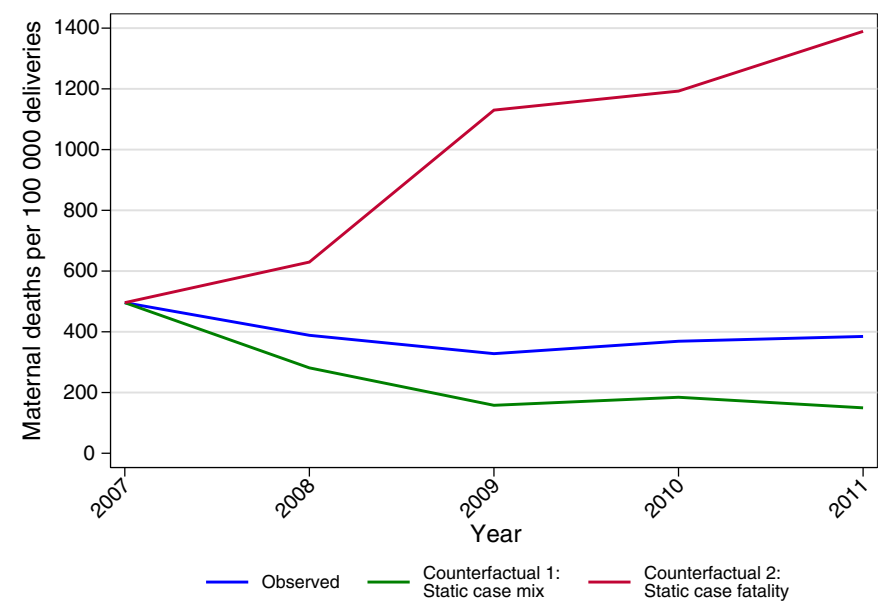

Fig. 1. Maternal mortality at Ridge Regional Hospital, 2007-2011, with counterfactua scenarios. 
implementation scores (both individual and combinations). Further, a second set of similarly constructed OLS regressions was performed using staff numbers and ratios by specialty and role (for anesthesia, midwifery, and obstetrics, and all staff combined). To mitigate against type I error, and because six groups were analyzed, a Bonferroni correction was implemented with a more restrictive $P$ value for multiple comparisons of $0.05 / 6=0.0083$.

To explore potential confounding between CQI activities and staffing, a third set of OLS regressions was performed using the CQI and staffing factors with significant correlation and which were predictive of outcome (i.e. in the same direction as the outcome trend). Every possible combination was analyzed using OLS regression for the four outcomes: all deliveries, $\mathrm{OH}, \mathrm{HDoP}$, and CF1. The $P$ value for significance was $0.05 / 4=0.0125$ for these analyses.

\section{Results}

Maternal mortality at RRH decreased by $22.4 \%$ between 2007 and 2011, from 496 to 385 deaths per 100000 deliveries (Table 2). Progress was fastest in the first program year. Significant changes occurred in delivery volume and case severity. Deliveries increased from 6049 in 2007 to 9357 in 2011 (Table 2). The proportion of pregnancies complicated by $\mathrm{OH}$ increased from $0.9 \%$ (95\% confidence interval [CI] $0.7 \%-1.1 \%$ ) in 2007 to $5.2 \%$ (95\% Cl 4.8\%-5.7\%) in 2011. Likewise, HDoP increased from $5.3 \%$ (95\% CI $4.7 \%-5.9 \%$ ) in 2007 to $14.5 \%$ (95\% CI $13.8 \%-15.2 \%$ ) in 2011.

Without adjusting for severity, the overall change in maternal mortality was not significant $\left(P=0.30\right.$; two-tailed $\chi^{2}$ with Yates correction); however, CFRs for the major obstetric complications decreased significantly. The CFR for $\mathrm{OH}$ decreased from $14.8 \%$ to $1.6 \%$ $(P<0.001)$ and that for HDoP from 3.1\% to $1.1 \%(P=0.0154)$ over the study period. Had there been no increase in complicated pregnancies, as depicted in case scenario CF1, maternal mortality would have dropped to 150 per 100000 deliveries $(P<0.001)$. Comparing CF2 with the observed maternal mortality ratio (MMR), it is estimated that a total of 245 maternal deaths were averted, including 94 in 2011 alone (Table 2).

Implementation of CQI activities was monitored over 5 years. By the project's end, 30 items were fully implemented, 33 were implemented by $75 \%, 12$ were implemented by $50 \%, 16$ were implemented by $25 \%$, and six items were not implemented (Fig. 2; individual implementation scores are shown in Supplementary Material S1). The overall implementation score was $68 \%$. The P bundle had the highest implementation (72\%), followed by the $\mathrm{Q}(68 \%)$ and the $\mathrm{S}(65 \%)$ bundles.

A list of significant CQI activities and staffing associated with CFR reductions was compiled (Supplementary Material S1). In general, staffing ratios were not predictive of improvement; however, the number of anesthesia nurses and labor-ward midwives correlated with CFR reductions for both complicated and uncomplicated deliveries.

Two categories from the Framework of Change Model-the S and Q1 bundles-stood out as being significant predictors of improvement in $\mathrm{HDoP}, \mathrm{OH}$, and CF1. The S bundle and subgroups Q3, S1, and S2 were all correlated with survival in cases of HDoP. Two of the most significant findings were from individual items Q2-6 (high-risk patients should be referred to the doctor within 30 min of midwife triage) and Q3-3 (host quarterly mortality conference for information dissemination to stakeholders). For $\mathrm{OH}$, mean implementation scores for all $\mathrm{CQI}$ activities, the $\mathrm{Q}, \mathrm{S}$, and P bundles, as well as all sub-bundles (except P1) were significantly correlated with improved survival. Similarly, the number of prenatal and labor midwives, obstetric house staff, and anesthesia nurses also significantly correlated with improved survival for $\mathrm{OH}$.

\section{Discussion}

Evaluating the effectiveness of complex interventions to reduce maternal mortality in low-income countries is challenging. In the absence of randomized control trials, quasi-experimental pre- and post-implementation designs are useful when pre-intervention outcome variables are known. The present analysis found that implementation of a CQI program was accompanied by a $22.4 \%$ reduction in maternal mortality from 2007 to 2011. This improvement occurred despite a nearly $50 \%$ increase in annual deliveries and five-fold and three-fold increases in the proportion of deliveries complicated by $\mathrm{OH}$ and HDoP, respectively.

For referral institutions, the MMR could be an unreliable indicator of performance because of changes in case mix or case severity. This measure alone may overestimate or underestimate the change that actually occurred. To begin with, each institution has a fairly small sample size,

Table 2

Delivery trends and counterfactual analysis.

\begin{tabular}{|c|c|c|c|c|c|}
\hline Variable & 2007 & 2008 & 2009 & 2010 & 2011 \\
\hline Total deliveries & 6049 & 7465 & 8230 & 8133 & 9357 \\
\hline Prevalence of $\mathrm{OH}, \%$ & 0.9 & 1.3 & 3.9 & 4.2 & 5.2 \\
\hline Prevalence of HDoP, \% & 5.3 & 7.8 & 12.1 & 12.7 & 14.5 \\
\hline Death rate from $\mathrm{OH}\left(\mathrm{CFR}_{\mathrm{hem}}\right), \%$ & 14.8 & 5.1 & 1.9 & 2.0 & 1.6 \\
\hline Death rate from $\mathrm{HDoP}\left(\mathrm{CFR}_{\mathrm{htn}}\right), \%$ & 3.1 & 1.4 & 1.1 & 1.1 & 1.1 \\
\hline Death rate from all others $\left(\mathrm{CFR}_{\mathrm{other}}\right)$, per 1000 & 0.21 & 0.24 & 0.14 & 0.18 & 0.17 \\
\hline \multicolumn{6}{|l|}{ Observed mortality statistics } \\
\hline Observed death rate ( CFR $\left._{\text {total }}\right)$, per 100000 deliveries & 496 & 388 & 328 & 369 & 385 \\
\hline Observed maternal deaths (total) & 30 & 29 & 27 & 30 & 36 \\
\hline Annual rate of change from baseline $\left(\mathrm{CFR}_{\text {total }}\right), \%$ & - & -24.4 & -20.7 & -9.9 & -6.3 \\
\hline Cumulative change from baseline $\left(\mathrm{CFR}_{\text {total }}\right), \%$ & - & -21.7 & -33.9 & -25.6 & -22.4 \\
\hline \multicolumn{6}{|l|}{ Mortality statistics assuming unchanged case mix from 2006 to 2011 (CF1) } \\
\hline Death rate (CFR-CF1), per 100000 deliveries & 496 & 281 & 158 & 184 & 150 \\
\hline Total maternal deaths expected (CF1) & 30 & 21 & 13 & 15 & 14 \\
\hline Annual rate of change from baseline (CFR-CF1), \% & - & -56.7 & -57.2 & -33.0 & -30.0 \\
\hline Cumulative change from baseline (CFR-CF1), \% & - & -43.3 & -68.2 & -62.8 & -69.8 \\
\hline \multicolumn{6}{|c|}{ Mortality statistics assuming unchanged case fatality from 2006 to 2011 (CF2) } \\
\hline Death rate (CFR-CF2), per 100000 deliveries & 496 & 630 & 1130 & 1193 & 1389 \\
\hline Total maternal deaths expected (CF2) & 30 & 47 & 93 & 97 & 130 \\
\hline Annual rate of change from baseline (CFR-CF2), \% & - & 23.9 & 41.2 & 29.2 & 25.8 \\
\hline Cumulative change from baseline (CFR-CF2), \% & - & 26.9 & 127.8 & 140.5 & 180.1 \\
\hline \multicolumn{6}{|l|}{ Mortality reduction and deaths averted } \\
\hline Death rate reduction from baseline (observed) & - & 107 & 168 & 127 & 111 \\
\hline Annual deaths averted (observed) & - & 8 & 14 & 10 & 10 \\
\hline Annual deaths averted with severity adjustment (CF2, observed) & - & 18 & 66 & 67 & 94 \\
\hline
\end{tabular}

Abbreviations: $\mathrm{OH}$, obstetric hemorrhage; HDoP, hypertensive disorders of pregnancy; $\mathrm{CFR}$, case fatality rate; $\mathrm{CF} 1$, counterfactual 1; CF2, counterfactual 2. 

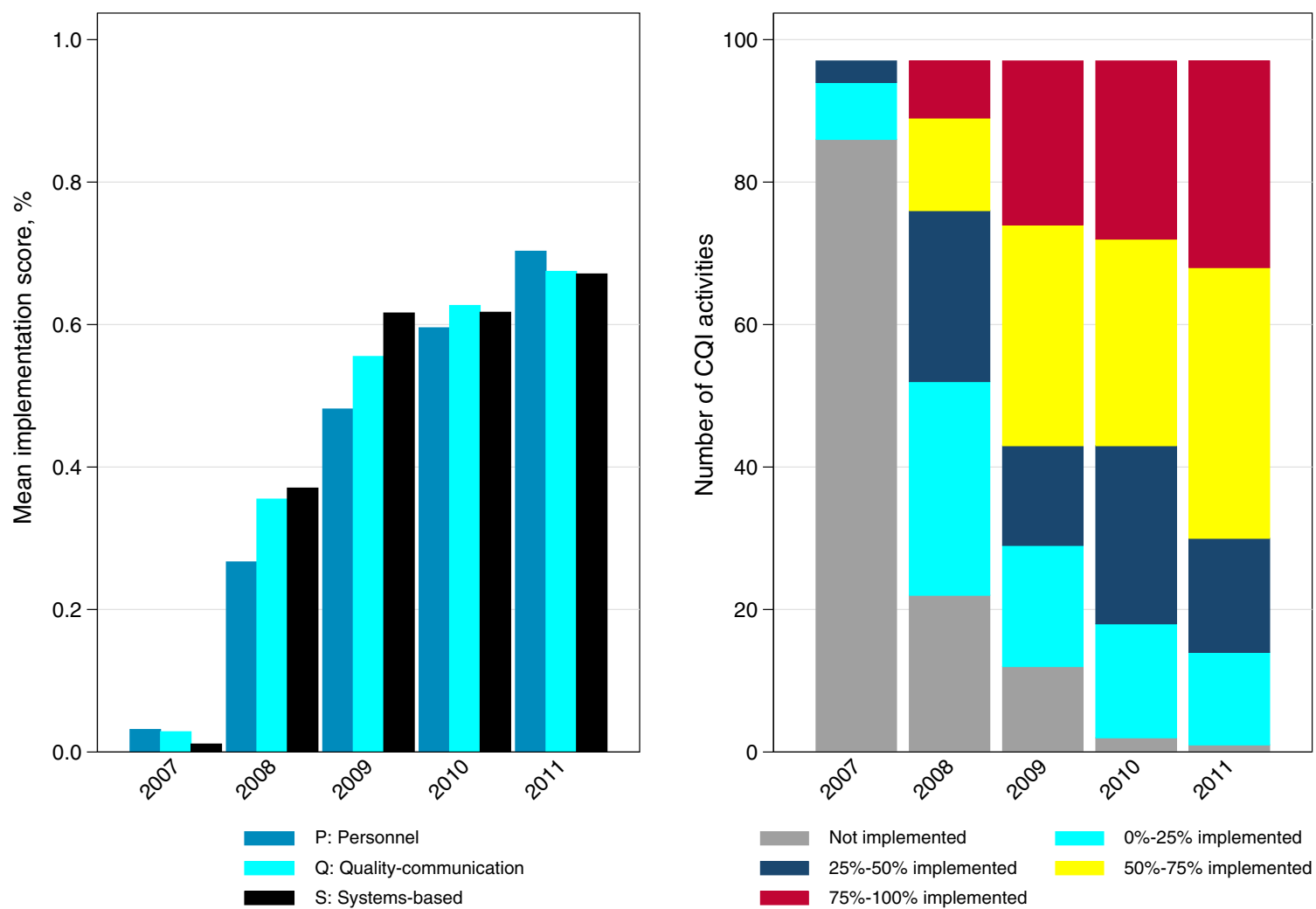

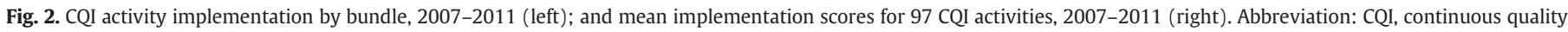
improvement.

making a metric measured on the order of per 100000 live births highly variable because small changes in the absolute number of deaths could lead to amplified changes in the MMR. Furthermore, increases in the proportion of complicated cases and volume could lead to a worsening of MMR even with an intervention. With the inception of this project, and through information dissemination, RRH became a preferred destination for high-risk referrals. The MMR cannot tell the entire story when a higher proportion of high-risk cases are increasingly referred.

When the increase in complicated deliveries was accounted for, the clinical improvements were more impressive: a near 70\% maternal mortality reduction over 5 years. Without the CQI program, maternal mortality at RRH could have been 1389 deaths per 100000 deliveries, consistent with the MMRs of 957-1004 reported concurrently in other regional and teaching hospitals in Ghana $[12,13]$. Additionally, the correlation between maternal mortality and CQI implementation supports a role for process-oriented efforts to improve clinical performance. The success of this partnership is consistent with projects in other West African referral hospitals, where focus on best practice implementation, early referral, and externally facilitated training saw greater mortality reductions than in centers without such programs [3].

With respect to staffing, it is no coincidence that the available numbers of labor midwives and nurse anesthetists were correlated with improvement, because both provide important lifesaving functions [14-17]. The worsening staffing ratios for many provider groups in this report are concerning. An increasing workload can be detrimental to morale, workplace health, attentiveness, and outcome [18]. One model described that a midwife could conduct an average of 175 births per year and that six midwives would be required for approximately 1000 births [19]. Hospitals providing secondary and tertiary levels of care have, by definition, a larger proportion of women needing such levels of care and require a higher number of midwives per 1000 deliveries. Ghana has a national average of 10.9 midwives per 1000 deliveries; in the Greater Accra Region, where RRH is located, there were 16.3 midwives per 1000 deliveries. Nevertheless, RRH had merely 6.6-7.1 midwives per 1000 deliveries per year, suggesting a misdistribution of midwives in the region. Thus, better staffing ratios could have further contributed to improved care. These results indicate that there is a need for redistribution of midwives on the basis of workload analysis. No similar figure exists for anesthesia or obstetric personnel. Obstetrician input has been shown to reduce cesarean deliveries and improve perinatal outcomes [20]. Indeed, there is a need for obstetrician involvement in the labor ward in response to increasing case-mix complexity and intervention rates. In Ghana, the national target for obstetricians is 455 ; however, there are only 279 at post, with $40 \%$ being in the public sector. Determining optimum staffing ratios is an area warranting further research.

The large number of correlated CQI activities underscores the multifactorial nature of improving survival in a rapidly changing, complex environment complicated by $\mathrm{OH}$ and HDoP, and supports the concept put forward in 2006 by The Lancet Maternal Survival Series that no single process change can lead to sustained development unless the entire system improves [21]. Although the goal should be to prevent complications, investment in both process-based solutions and human resources is essential in creating a well-functioning health system capable of averting deaths. Although many activities were found to be important, the $\mathrm{S}$ bundle emerged as having the greatest impact on clinical outcome. This is not surprising because it contains activities aimed at addressing delay, shortages of supplies, equipment, and trained personnel, which are all often cited as contributing factors to maternal mortality [22,23].

The present analysis has some limitations. First, because no individual-level data were collected, it was impossible to analyze potential clinical confounders. Second, the intervention was aimed at optimizing emergency obstetric care and did not address factors associated with delays in seeking care [24]. Third, data collection for implementation occurred on a triannual basis, but maternal mortality statistics were tabulated annually, which limited the temporal precision of the correlation analysis and could have truncated the number of significant CQI activities. 
Fourth, correlation cannot prove causation in the present single-center study. Fifth, the structure of the correlation analysis-with $97 \mathrm{CQI}$ activities in nine sub-bundles and three bundles, 10 groups of clinical staff, and six outcomes-presented analytical challenges. The technique for measuring CQI implementation is a science in development, with few studies to date measuring the implementation rates of individual improvement activities [25]. The present study was able to qualitatively assess potential confounding between staffing and CQI activities, but not between the different activities.

In conclusion, this program involved the application of CQI and general institutional care strengthening methodologies to maternity services in a large regional referral hospital in Ghana. An evaluation framework was built, involving the continuous assessment of implementation of predetermined improvement activities, the calculation of outcomes using risk adjustment to determine two counterfactual scenarios, and the application of regression analysis to determine significant factors associated with improved performance. Finally, as the international community strives to match the most effective measures for reducing maternal mortality with the countries that need them, locally driven, comprehensive CQI programs must be considered.

Supplementary data to this article can be found online at http://dx. doi.org/10.1016/j.ijgo.2015.11.026.

\section{Acknowledgments}

Funding in support of the project was provided by the International Association for the Study of Pain, the Lacy Foundation, and the Obstetric Anaesthetists' Association.

\section{Conflict of interest}

A.J.O. and M.D.O. report grants from the Making Every Baby Count Initiative (September 2013). E.K.S, N.J.K., and D.M.G. have no conflicts of interest. Kybele, Inc. is the sub-recipient of a grant awarded to PATH (Program for Appropriate Technology in Health).

\section{References}

[1] Hussein J, Kanguru L, Astin M, Munjanja S. The effectiveness of emergency obstetric referral interventions in developing country settings: a systematic review. PLoS Med 2012;9(7), e1001264.

[2] Pucher PH, Macdonnell M, Arulkumaran S. Global lessons on transforming strategy into action to save mothers' lives. Int J Gynecol Obstet 2013;123(2):167-72.

[3] Dumont A, Fournier P, Abrahamowicz M, Traoré M, Haddad S, Fraser WD, et al. Quality of care, risk management, and technology in obstetrics to reduce hospital-based maternal mortality in Senegal and Mali (QUARITE): a cluster-randomised trial. Lancet 2013;382(9887):146-57.

[4] World Health Organization. Everybody's Business: Strengthening Health Systems to Improve Health Outcomes: WHO's Framework for Action. http://www.who.int/ healthsystems/strategy/everybodys_business.pdf. Published 2007. Accessed March 31, 2016.

[5] Nyamtema AS, Urassa DP, van Roosmalen J. Maternal health interventions in resource limited countries: a systematic review of packages, impacts and factors for change. BMC Pregnancy Childbirth 2011;11:30.

[6] Kayongo M, Butera J, Mboninyibuka D, Nyiransabimana B, Ntezimana A, Mukangamuje V. Improving availability of EmOC services in Rwanda-CARE's experiences and lessons learned at Kabgayi Referral Hospital. Int J Gynecol Obstet 2006;92(3):291-8.

[7] Srofenyoh E, Ivester T, Engmann C, Olufolabi A, Bookman L, Owen M. Advancing obstetric and neonatal care in a regional hospital in Ghana via continuous quality improvement. Int J Gynecol Obstet 2012;116(1):17-21.

[8] Svoronos T, Mate KS. Evaluating large-scale health programmes at a district level in resource-limited countries. Bull World Health Organ 2011;89(11):831-7.

[9] Wilson A, Hillman S, Rosato M, Skelton J, Costello A, Hussein J, et al. A systematic review and thematic synthesis of qualitative studies on maternal emergency transport in low- and middle-income countries. Int J Gynecol Obstet 2013;122(3):192-201.

[10] Peabody JW, Tozija F, Muñoz JA, Nordyke RJ, Luck J. Using vignettes to compare the quality of clinical care variation in economically divergent countries. Health Serv Res 2004;39(6 Pt 2):1951-70.

[11] Curran-Everett D, Williams CL. Explorations in statistics: the analysis of change. Adv Physiol Educ 2015;39(2):49-54.

[12] Lee QY, Odoi AT, Opare-Addo H, Dassah ET. Maternal mortality in Ghana: a hospitalbased review. Acta Obstet Gynecol Scand 2012;91(1):87-92.

[13] Ganyaglo GY, Hill WC. A 6-year (2004-2009) review of maternal mortality at the Eastern Regional Hospital, Koforidua, Ghana. Semin Perinatol 2012;36(1):79-83.

[14] Ouro-Bang'na Maman AF, Tomta K, Ahouangbévi S, Chobli M. Deaths associated with anaesthesia in Togo, West Africa. Trop Doct 2005;35(4):220-2.

[15] Vieira C, Portela A, Miller T, Coast E, Leone T, Marston C. Increasing the use of skilled health personnel where traditional birth attendants were providers of childbirth care: a systematic review. PLoS One 2012;7(10), e47946.

[16] Kane RL, Shamliyan TA, Mueller C, Duval S, Wilt TJ. The association of registered nurse staffing levels and patient outcomes: systematic review and meta-analysis. Med Care 2007;45(12):1195-204.

[17] Ashcroft B, Elstein M, Boreham N, Holm S. Prospective semistructured observational study to identify risk attributable to staff deployment, training, and updating opportunities for midwives. BMJ 2003;327(7415):584.

[18] Das JK, Kumar R, Salam RA, Lassi ZS, Bhutta ZA. Evidence from facility level inputs to improve quality of care for maternal and newborn health: interventions and findings. Reprod Health 2014;11(Suppl. 2):S4.

[19] UNFPA. The State of the World's Midwifery 2011. Delivering Health, Saving Lives. http://www.unfpa.org/publications/state-worlds-midwifery-2011. Published 2011. Accessed June 16, 2015.

[20] Browning A, Menber B. Reducing maternal morbidity and mortality in the developing world: a simple, cost-effective example. Int J Womens Health 2015;7:155-9.

[21] Campbell OM, Graham WJ, Lancet Maternal Survival Series steering group. Strategies for reducing maternal mortality: getting on with what works. Lancet 2006;368(9543): 1284-99.

[22] Wagaarachchi PT, Graham WJ, Penney GC, McCaw-Binns A, Yeboah Antwi K, Hall $\mathrm{MH}$. Holding up a mirror: changing obstetric practice through criterion-based clinical audit in developing countries. Int J Gynecol Obstet 2001;74(2):119-30.

[23] Weeks AD, Alia G, Ononge S, Otolorin EO, Mirembe FM. A criteria-based audit of the management of severe pre-eclampsia in Kampala, Uganda. Int J Gynecol Obstet 2005;91(3):292-7.

[24] Barnes-Josiah D, Myntti C, Augustin A. The "three delays" as a framework for examining maternal mortality in Haiti. Soc Sci Med 1998;46(8):981-93.

[25] Leatherman S, Ferris TG, Berwick D, Omaswa F, Crisp N. The role of quality improvement in strengthening health systems in developing countries. Int J Qual Health Care 2010;22(4):237-43. 